\title{
Re: Could Testosterone Replacement Therapy in Hypogonadal Men Ameliorate Anemia, a Cardiovascular Risk Factor? An Observational, 54-week Cumulative Registry Study
}

\section{Zhang LT, Shin YS, Kim JY, Park JK}

Chonbuk National University and Research Institute of Clinical Medicine of Chonbuk National University-Biomedical Research Institute and Medical Device Clinical Trial Center, Department of Urology, Jeonju, Republic of Korea

J Urol 2016;195:1057-1064. doi: 10.1016/j.juro.2015.10.130. Epub 2015 Oct 28.

\section{EDITORIAL COMMENT}

Testosterone deficiency syndrome may associate with erectile dysfunction, increased abdominal fat and reduced muscle mass. Low serum testosterone is also related with anemia, metabolic syndrome and cardiovascular disease. In this study, the authors investigated if testosterone undecanoate (TU) reduces anemia and the risk of cardiovascular disease in patients with hypogonadism A total of 58 participants with a total testosterone level of less than $2.35 \mathrm{ng} / \mathrm{ml}$ received an injection of $1.000 \mathrm{mg}$ TU 6 times; at initial visit, 6, 18, 30, 42 and 54 weeks. They observed that total testosterone and free testosterone levels were restored by TU. Hemoglobin and hematocrit levels significantly increased while anemia and total cholesterol levels significantly reduced. Although there are some limitations of this study e.g. it is not a randomized controlled and a long-term study, TU treatment in hypogonadal men decreased the prevalence of anemia, improved lipid profiles and lowered the risk of cardiovascular disease.

Emre Bakırcıoğlu, MD

\section{Re: A Randomized Prospective Double-Blind Comparison Trial of Clomiphene Citrate and Anastrozole in Raising Testosterone in Hypogonadal Infertile Men}

\author{
Helo S1, Ellen J1, Mechlin C2, Feustel P3, Grossman M4, Ditkoff E4, McCullough A1 \\ ${ }^{1}$ Albany Medical Center, Clinic of Urology, New York, USA \\ 2 Urology Associates of Central Missouri, Missouri, USA \\ 3 Albany Medical College, Center for Neuroscience and Neuropharmacology, New York, USA \\ ${ }^{4}$ CNY Fertility Center, New York, USA
}

J Sex Med 2015;12:1761-1769. doi: 10.1111/jsm.12944. Epub 2015 Jul 14.

\section{EDITORIAL COMMENT}

It has been reported that the prevalence of low testosterone $(<300 \mathrm{ng} / \mathrm{ml})$ in infertile men was as high as $41 \%$ and low testosterone did not always correlate with impaired seminal parameters (1). Although previous studies have shown that clomiphene citrate (CC) and anastrozole (AZ) were effective in increasing testosterone levels, randomized double blind comparisons are lacking. In this study, the authors aimed to investigate the equivalence of CC versus AZ with respect to improvement in testosterone levels in hypogonadal infertile men. It was shown that there was a significantly larger increase in $\mathrm{T}$ level in CC group vs AZ group at 6 and 12 weeks whereas estradiol levels increased in CC group compared to AZ group. Both groups did not demonstrate significant changes in seminal parameters or patient-reported outcomes, This study shows that CC would be a better choice to increase testosterone levels for hypogonadal men with low estradiol levels.

\section{Reference}

1. Sigman M, Jarow JP. Endocrine evaluation of infertile men. Urology 1997;50:659-664. 